\title{
Herramientas tecnológicas para la gestión de información científica en estudiantes de la carrera de Agronomía
}

\section{Technological tools for the management of scientific information in students of the Agronomy career}

\section{Herramientas tecnológicas para la gestión de información científica}

Juan Pío Salazar Arias ${ }^{(1)}$

Tatiana Carolina Gavilánez Buñay ${ }^{(2)}$

Wellington Jean Picay Ronquillo(3)

Cristian Santiago Tapia Ramírez ${ }^{(4)}$

(1) Facultad de Ciencias Agropecuarias y Recursos Naturales. Ecuador. email: juan.salazar0@utc.edu.ec. ORCID: http://orcid.org/0000-0002-1609-0085

(2) Facultad de Ciencias Agropecuarias y Recursos Naturales. Ecuador. email: tatiana.gavilanez@utc.edu.ec . ORCID:http://orcid.org/0000-0002-7422-3122

(3) Facultad de Ciencias Agropecuarias y Recursos Naturales. Ecuador. email: wellington.pincay4586@utc.edu.ec. ORCID: http://orcid.org/0000-0003-3366-6477

(4) Facultad de Ciencias Agropecuarias y Recursos Naturales. Ecuador. email: cristian.tapia4416@utc.edu.ec. ORCID: http://orcid.org/0000-0003-2104-5972

Contacto: juan.salazar0@utc.edu.ec

\section{Recibido: 15-11-2020 Aprobado: 10-12-2020}

\section{Resumen}

La educación ambiental constituye una opción vital ante la crisis en que se halla inmerso el mundo, de ahí la importancia de las asignaturas que forman cultura y consciencia en estos temas. En el presente trabajo se realiza un análisis del empleo de las herramientas de infotecnología en la gestión de información en los estudiantes que cursan la asignatura Educación Ambiental en la carrera Agronomía, 
de la Universidad Técnica de Cotopaxi, en Ecuador. La población objeto de estudio se encuentra conformada por 37 estudiantes y se seleccionó una muestra de 33 alumnos. La investigación se desarrolló bajo un enfoque cuantitativo de tipo exploratorio descriptivo, a partir de un diseño no experimental descriptivo. Se obtiene un diagnóstico que permite proyectar la formación de competencias tecnológicas que apoyen el desarrollo de los proyectos de investigación de los estudiantes.

Palabras claves: gestión de información, infotecnología, carrera de Agronomía

\begin{abstract}
Environmental education constitutes a vital option in the face of the crisis in which the world is immersed, hence the importance of the subjects that form culture and awareness on these issues. In this work, an analysis of the use of infotechnology tools in information management is carried out in students who take the Environmental Education subject in the Agronomy career, at the Technical University of Cotopaxi, in Ecuador. The study population is made up of 37 students and a sample of 33 students was selected. The research was developed under a descriptive exploratory quantitative approach, based on a descriptive non-experimental design. A diagnosis is obtained that allows projecting the formation of technological competencies that support the development of student research projects.
\end{abstract}

Keywords: information management, infotechnology, agronomy career

\title{
Introducción
}

En la actualidad el desarrollo de las Tecnologías de la Información y la Comunicación (TIC), han generado grandes volúmenes de información en cualquier área del conocimiento, incluyendo las Ciencias Agrícolas. En este sentido Wilson et al. (2013), plantean que la utilización de esta información se dificulta a medida que crece y se hace ineludible el empleo de herramientas tecnológicas que sean eficaces para la búsqueda y gestión de información relevante para los estudiantes.

Moncada (2014), afirma que por lo general un porcentaje alto de estudiantes universitarios optan por emplear la herramienta Google como la primera opción de obtención de información en actividades académicas y por lo general los resultados que se alcanzan no son favorables. También asevera la 
necesidad de desarrollar habilidades en los educandos para la búsqueda de fuentes bibliográficas válidas.

Gómez et al. (2014), hacen referencia a la importancia de contar con estrategias de búsqueda, organización y análisis de la información, pues permiten la obtención de la información que sirve de referente a un tema de investigación. Los autores aseveran queel trabajo de revisión de la literatura científica y académica constituye una etapa fundamental de todo proyecto de este tipo y debe garantizar la obtención de la información más relevante en el campo de estudio.

En este sentido Reyes et al. (2020), plantean la necesidad de crear estrategias para fortalecer el conocimiento y el uso de herramientas tecnológicas, de manera que se conviertan en un hábito del proceso investigativo de los estudiantes universitarios en la Universidad Técnica de Cotopaxi, en Ecuador. Estos autores refieren la necesidad de fortalecer la preparación de los estudiantes para que puedan lograr las habilidades necesarias en el uso de las herramientas infotecnológicas que les permita reforzar la cultura investigativa en la carrera de Agronomía.

El presente trabajo tiene por objetivo analizar el empleo de las herramientas de infotecnología en la gestión de información en los estudiantes que cursan la asignatura Educación Ambiental en la carrera Agronomía, en la Universidad Técnica de Cotopaxi, en Ecuador.

\section{Material y métodos}

La investigación se ejecutó en la Universidad Técnica de Cotopaxi. La población objeto de estudio, se encuentra conformada por 37 estudiantes de la carrera Agronomía y que cursan la asignatura de Educación Ambiental. La muestra estuvo conformada por 33 alumnos.

Para la recolección de la información se utilizó la técnica de encuesta no estructurada. Y como instrumento se empleó el cuestionario, que respondieron los 33 educandos que participaron en la investigación y al que se pudo acceder en formato digital, el mismo contó con cinco preguntas que permitieron evaluar el uso de las herramientas infotecnológicas para la gestión de información y el desarrollo de proyectos de investigación en la asignatura Educación Ambiental en la carrera Agronomía, en la Universidad Técnica de Cotopaxi. 
La investigación se desarrolló bajo un enfoque cuantitativo. El tipo de investigación fue exploratorio descriptivo, a partir de un diseño no experimental descriptivo. Una vez obtenidas las respuestas del cuestionario se procedió a tabularlas mediante el tabulador electrónico Excel y se diseñaron gráficos con las respuestas agrupadas por cada ítem.

\section{Desarrollo}

El principal reto en la gestión ambiental, está en alcanzar una estrategia compatible, de manera que se garantice un desarrollo sostenible, involucrando a todos los entes y actores sociales territoriales $\mathrm{y} / \mathrm{o}$ sectoriales, buscando la implementación de escenarios de integración de la dimensión ambiental con otras políticas de producción: agraria, pesquera, industrial, educacional, entre otras, como forma de manejo sistémico e integrador de todo el patrimonio. Según Ayes (2004), las deficiencias que se han presentado en varios países han tenido como causa, fundamentalmente, la insuficiente conciencia, conocimientos y educación ambiental, la carencia de una mayor exigencia en la gestión, la insuficiente incorporación de la dimensión ambiental en las políticas, planes y programas de desarrollo y por la ausencia de un sistema jurídico lo suficientemente integrador y coherente.

Los profesionales de las Ciencias Agrícolas en el siglo XXI y, con más razón en el contexto actual de riesgo epidemiológico, además de ser idóneos en sus disciplinas, deben ser capaces de buscar, de gestionar información novel y relevante, evaluarla con espíritu crítico, trabajar en equipos interdisciplinarios, estar familiarizados con videoconferencias, flexibilidad en la adopción del uso de aplicaciones informáticas para el manejo y gestión de información y las herramientas innovadoras (por ejemplo tractores sin operarios, drones para uso en agricultura y herramientas digitales para monitoreo de plagas y enfermedades), además de un entorno laboral sumamente competitivo.

No obstante, Flores (2019), plantea que en muchas de las actividades formativas de estos futuros profesionales, se observa aún el uso de tecnologías alejadas de los requerimientos demandados por el mercado laboral actual. Entre ellos se puede citar el uso restringido de planillas electrónicas de cálculo a favor de calculadoras de mano, lápiz y papel y el uso de herramientas no informáticas para el cálculo de áreas (planímetros) en lugar de programas específicos para ordenadores (Sistemas de Información Geográfica o SIG), tan sólo por citar algunos ejemplos. 


\section{Resultados}

Al aplicar la encuesta se comprobó que el 78\% (26 estudiantes) usaban en ocasiones herramientas tecnológicas para gestionar información relacionada con los contenidos de la asignatura Educación Ambiental, solo el 12\% (4) las utilizaban siempre. Manifestaron, además, emplear en diversa medida otras fuentes bibliográficas, primando el uso de los materiales preparados por los docentes de la asignatura para guiar el estudio (Gráfico 1).

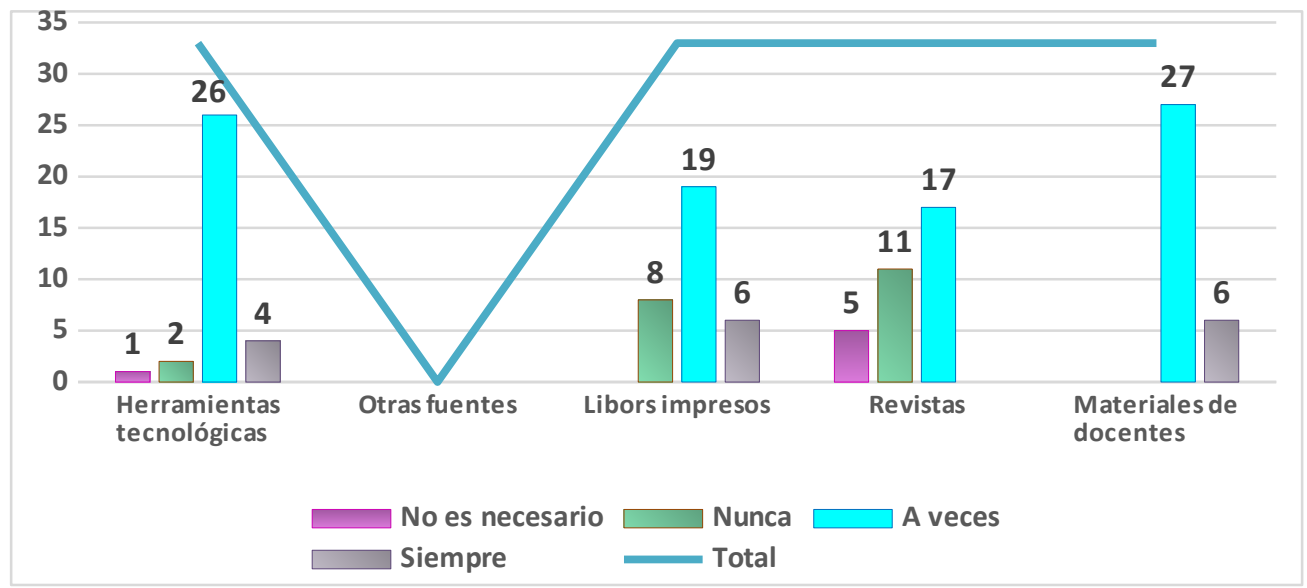

Gráfico 1. Uso de herramientas tecnológicas y otras fuentes para gestionar información.

Los 30 estudiantes que refieren diferentes grados de uso de las herramientas tecnológicas, expresan que Google, es el buscador más utilizado y solo 3 han empleado ocasionalmente a Bing. No tienen conocimiento sobre los metabuscadores, directorios de materias y bases de datos, ni el impacto que estos tienen en la búsqueda de información científica en educación ambiental, válida y actualizada, que apoye los trabajos investigativos desarrollados en la asignatura.

Los docentes, al analizar los resultados del diagnóstico anterior, diseñan una preparación extracurricular para contribuir a formar en los alumnos las competencias infotecnológicas necesarias para cumplir los objetivos de la asignatura en particular y de los estudiantes como futuros egresados y profesionales del siglo XXI. Se impartieron conferencias - talleres para introducir las herramientas de búsqueda de información científica y laboratorios donde los alumnos gestionaban información relacionada con sus proyectos investigativos, usando las herramientas aprendidas.

Los resultados se pudieron comprobar en la calidad de los trabajos investigativos de la asignatura, en las fuentes bibliográficas utilizadas y en la actualidad de las mismas, además de ofrecerles a los 
estudiantes la posibilidad de confrontar sus resultados con investigaciones similares a nivel mundial y regional, ya que las temáticas de medio ambiente son una prioridad a nivel global.

Se utilizó, además, un cuestionario para determinar el nivel de satisfacción y uso de las herramientas aprendidas. En el gráfico 2 se muestra que el empleo de las herramientas varió en dependencia de la etapa del proyecto que se desarrollaba, lo que demuestra que los alumnos adquirieron adecuadamente la competencia; en la etapa de familiarización con el tema de educación ambiental que debían desarrollar, usaron Google, buscador que todos dominaban, y comenzaron a usar Scholar Google en un 96,96\% (32 estudiantes), seguido de ScienceResearh con un 42,42\%. Se destaca que en la justificación del problema y, por ende, la definición de los objetivos a cumplir en el proyecto, el 100\% usa un buscador académico como Scholar Google y el resto de las herramientas especializadas de Internet. Para contrastar los resultados alcanzados con los de otras investigaciones similares, se comprueba un balance entre los buscadores especializados y la Base de Datos Scielo.

Se evaluó además, el conocimiento y uso de herramientas tecnológicas en el proceso de consulta, colaboración y confrontación de los resultados alcanzados en el proyecto de investigación con otros especialistas, condiscípulos y docentes (Gráfico 3). Como se puede apreciar el 90,90\% (30 estudiantes) reconoce que nunca las ha usado y el resto considera que no es necesario.

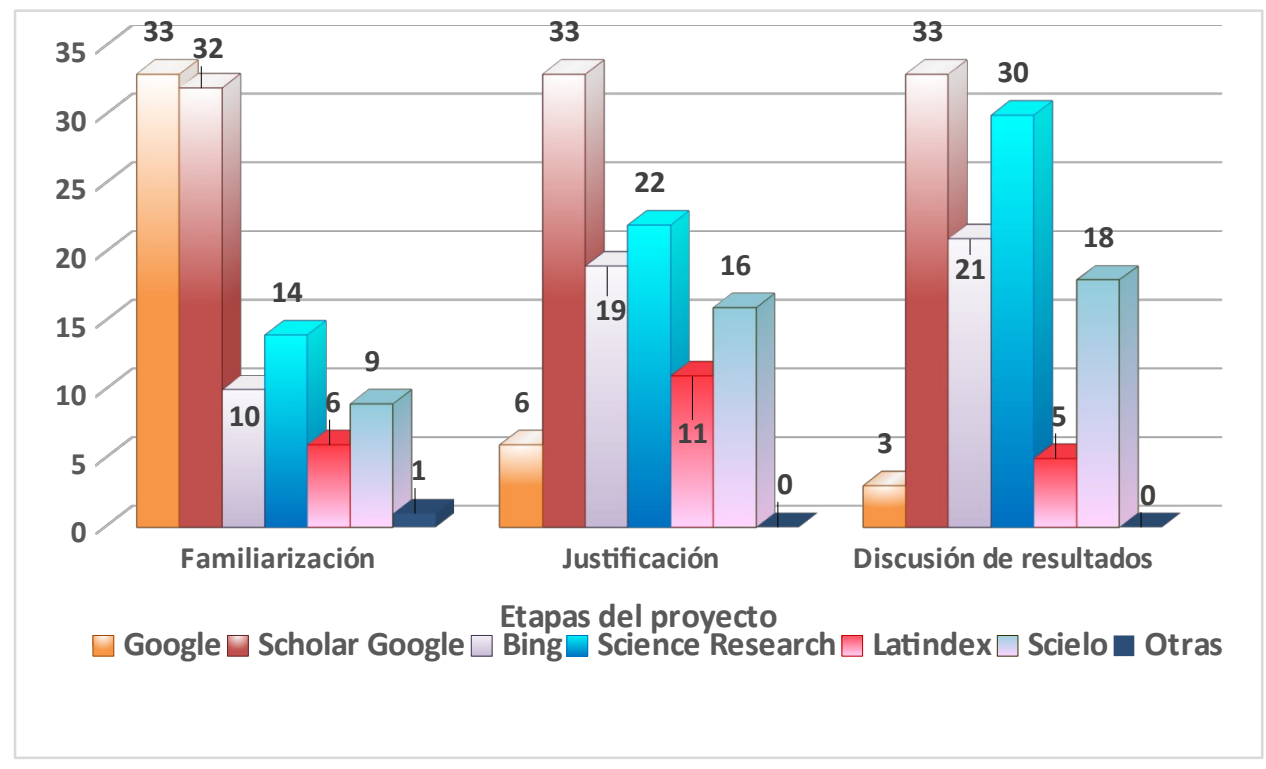

Gráfico 2. Empleo de las herramientas tecnológicas en el proyecto de la asignatura Educación Ambiental. 


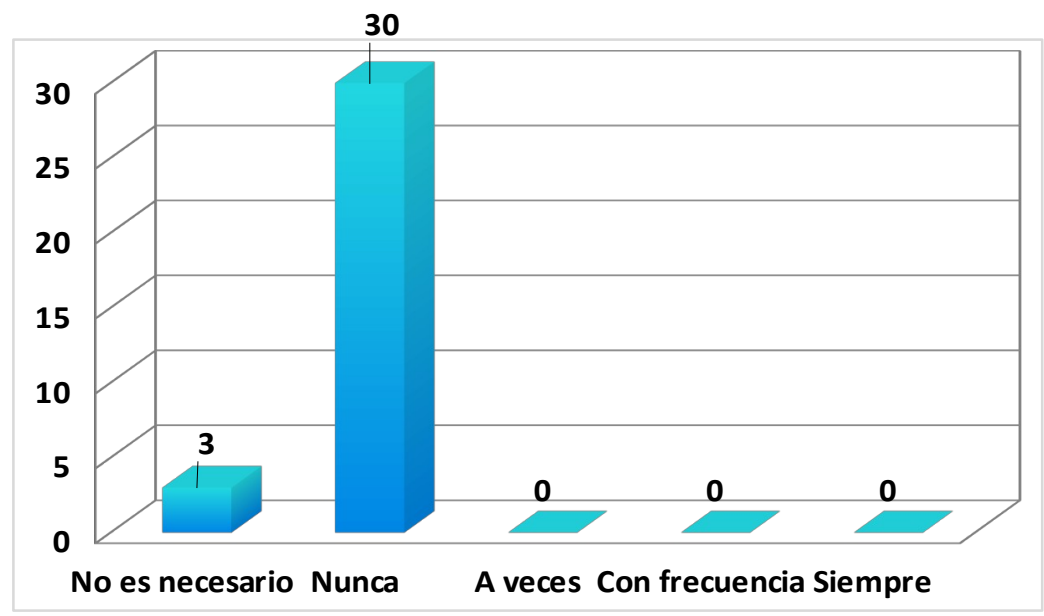

Gráfico 3. Uso y dominio de las herramientas tecnológicas en el proceso de confrontación científica

Los docentes analizaron también el grado de complejidad que los alumnos detectaron en el uso de cada una de las herramientas utilizadas, comprobándose que el uso de las Bases de Datos Latindex y Scielo tuvieron un grado de dificultad mayor, debido fundamentalmente, a que cambia la filosofía de búsqueda tradicional a la que los alumnos estaban acostumbrados (Gráfico 4).

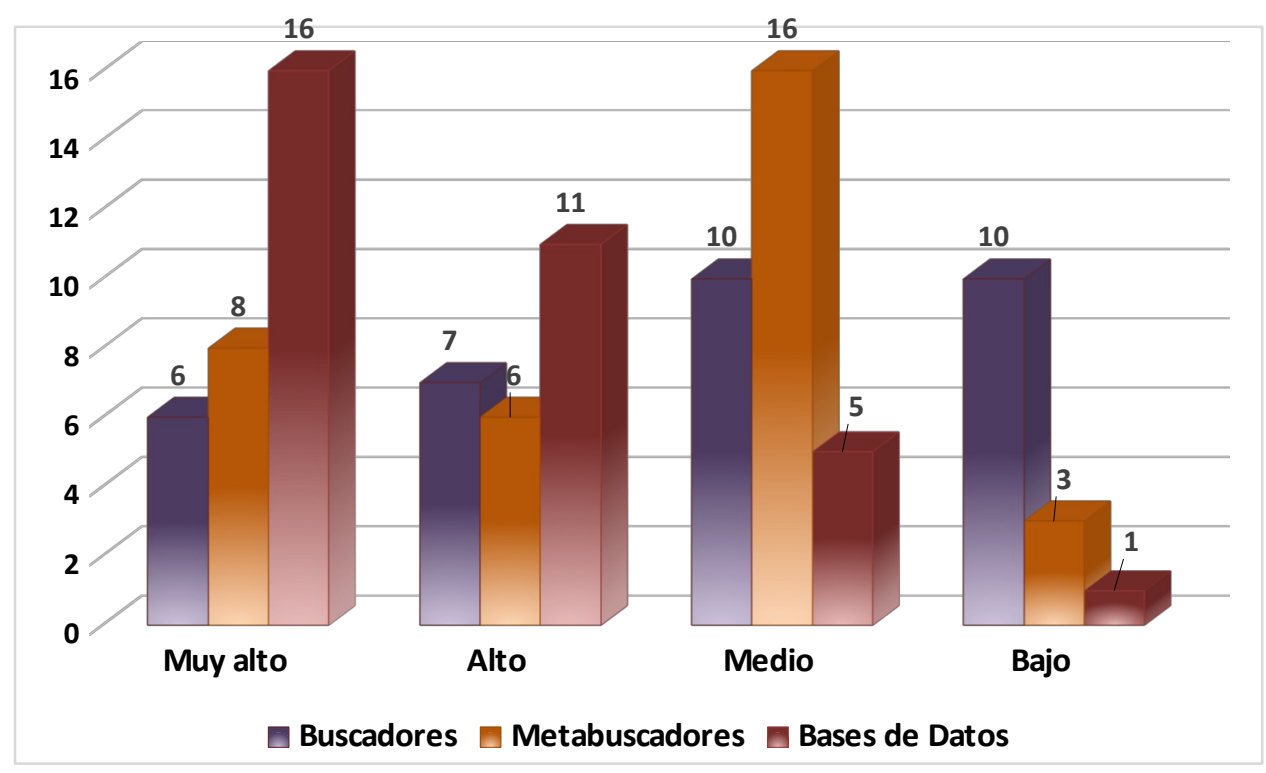

Gráfico 4. Nivel de complejidad definida por los estudiantes para cada herramienta tecnológica

Estos resultados le permiten a los docentes y guías de los proyectos de la asignatura Medio Ambiente, continuar profundizando en los temas y herramientas que la preparación de sus estudiantes para próximas etapas de formación.

\section{Discusión}


Para Freire (1995), la educación contribuye a una conciencia crítica e integral de nuestra situación en el planeta. También, es un agente importante en la transición a una nueva fase ecológica de la humanidad. Pretende comprender su relación en la biosfera humanizada, al formar personas capaces de interpretar y transformar el mundo, y de dar importancia a los derechos de todos los seres vivos (incluyendo humanos) y la naturaleza, para contribuir a plantear políticas y culturas basadas en necesidades a corto plazo. Coincidiendo con este autor, se desarrolla el proceso de enseñanza-aprendizaje de la asignatura Educación Ambiental en la carrera Agronomía, en la Universidad Técnica de Cotopaxi, en Ecuador. La presente investigación responde a la necesidad de formar competencias digitales que apoyen una gestión ambiental acorde a los tiempos actuales.

En este trabajo se coincide con Sarango et al. (2016), cuando plantean que la educación ambiental debe "sensibilizar, concientizar y motivar a individuos, entidades, organizaciones y sociedad en general acerca de la necesidad de no solo cuidar el medio ambiente, de vivir en armonía con él, sino de impulsarlos a la acción: crear la necesaria conciencia ambiental” (pág. 185). Esto alcanza una importancia vital en las instituciones educativas en todos los niveles de enseñanza. En las universidades es necesario preparar a los futuros profesionales para que adquieran las competencias de gestionar información que les permita tener en cuenta el cuidado del medio ambiente en sus puestos de trabajo y en la función que realicen, usando las herramientas que la sociedad de la información pone en sus manos.

Se concuerda con la Secretaría De Ambiente Y Desarrollo Sustentable (2009), cuando considera que la Formación Ambiental es un campo de conocimiento complejo y en construcción, en el cual se sostienen profundos debates epistemológicos y pedagógicos. En su investigación indaga sobre el sentido de los procesos educativos ambientales con el fin de que éstos, en el contexto de las prácticas, se constituyan en procesos tendientes al cambio social.

La motivación y los resultados de este trabajo se corresponden con lo planteado por los autores anteriores cuando plantean que para lograr la alfabetización y profesionalización ambiental es necesaria una revisión de los procesos de formación profesional en todas las disciplinas de conocimiento, incluyendo particularmente la formación docente y de educadores ambientales como facilitadores para la construcción de nuevos saberes y compromisos ciudadanos. 
Nuestro trabajo se corresponde con lo planteado en el Manifiesto por la vida: por una ética para la sustentabilidad de Galano et al. (2002), el que se plantea que;

La educación para la sustentabilidad debe entenderse en este contexto como una pedagogía basada en el diálogo de saberes, y orientada hacia la construcción de una racionalidad ambiental. Esta pedagogía incorporaunavisiónholísticadelmundoyunpensamientodelacomplejidad.Perovamásalláalfundarseenunaétic ayunaontologíadelaotredadquedelmundocerradodelasinterrelacionessistémicasdelmundoobjetivadode lo ya dado, se abre hacia lo infinito del mundo de lo posible y a la creación de "lo que aún noes". Es la educación para la construcción de un futuro sustentable, equitativo, justo y diverso. Es una educación para la participación, la autodeterminación y la transformación; una educación que permita recuperar el valor de lo sencillo en la complejidad; de lo local antelo global; de lo diverso ante lo único; de lo singular antelo universal (pág. 6 y 7).

Se coincide, además, con la Unesco (2004), cuando define que la educación ambiental puede y debe ser un factor estratégico que incida en el modelo de desarrollo establecido para reorientarlo hacia la sustentabilidad y la equidad. "Para contribuir con eficacia a mejorar el ambiente, la acción de la educación debe vincularse con la legislación, las políticas, las medidas de control y las decisiones que los gobiernos adopten, en relación con el ambiente humano" (UNESCO, 2004, s. p.).

Este propósito incluye, obviamente, el uso de herramientas especializadas que permitan crear comunidades de aprendizaje e investigación, de gestión y colaboración, en un mundo interconectado, por lo que se confirma la necesidad de crear las competencias en estudiantes de las carreras de Ciencias Agrícolas en el uso de las tecnologías adecuadas que constituyan un medio para proteger y desarrollar una cultura de protección al medio ambiente.

Para Martínez (2010), la educación ambiental debe ser un proceso que genere aprendizajes mediante la construcción y reconstrucción de conocimientos, como resultado del estudio de las complejas interacciones sociedad-ambiente, lo que ha de generar conciencia en la ciudadanía de su papel como parte integrante de la naturaleza, para que desarrollen nuevas relaciones, sentires, actitudes, conductas y comportamientos hacia ella. También valora los estilos de desarrollo y de progreso convencional, que se basan en la degradación de las aguas, de la tierra, del aire y no resuelve los problemas sociales (pobreza, miseria, concentración de riquezas, violencia y otras). Esta es también la óptica con la que en el presente trabajo se busca formar las competencias y la cultura ambiental en los estudiantes de la asignatura objeto de análisis. 
Para Rojas et al. (2003), la Educación Ambiental no es una asignatura aislada en el currículo, sino que debe concebirse como un eje transversal, la misma debe considerar las costumbres y las culturas locales, vincularse a situaciones vivenciales o de coordinación con otros sectores para promover actividades a favor del medio ambiente, responder a políticas institucionales contempladas por organizaciones gubernamentales, no gubernamentales y de educación no formales. Coincidiendo con estos autores, en la presente investigación las prácticas pedagógicas se orientan a la toma de conciencia sobre el deterioro ambiental, la protección y mejora del ambiente en zonas rurales y urbanas, usando las herramientas tecnológicas que garanticen la validez y actualidad de las fuentes consultadas.

Por último, la Unesco Etxea (2020), considera que el desarrollo sostenible, más que una meta a la que llegar, es un proceso, y que, por lo tanto, no se avanza sólo mediante la aplicación de técnicas y programas. Como todo proceso social, depende de los valores y formas de comportamiento humano. Esta realidad confiere a la educación, y más concretamente a la educación para la sostenibilidad y el medio ambiente, una función estratégica. Esto es, en definitiva, lo que persigue la 'Década de las Naciones Unidas de la Educación para el Desarrollo Sostenible' Unesco (2014), cuyo objetivo global consiste en "integrar los valores inherentes al desarrollo sostenible en todas las facetas del aprendizaje con vistas a fomentar los cambios de comportamiento necesarios para lograr una sociedad más sostenible y justa para todos" (pág. 4). Estos presupuestos confirman los resultados alcanzados en la presente investigación y las proyecciones futuras.

\section{Conclusiones}

Se realiza un análisis del empleo de las herramientas de infotecnología en la gestión de información en los estudiantes que cursan la asignatura Educación Ambiental en la carrera Agronomía, de la Universidad Técnica de Cotopaxi, en Ecuador. Se comprueba un bajo dominio de las herramientas tecnológicas factibles de usar en todas las etapas del desarrollo del proyecto de investigación de los estudiantes. Los resultados obtenidos permiten proyectar y rediseñar la formación de competencias tecnológicas que apoyen el desarrollo de los proyectos de investigación de los estudiantes.

\section{Bibliografía}

Ayes, G. (2004). Medio ambiente: Impacto y desarrollo (Vol. Divulgación Científica). Ciudad de La Habana: Científico-Técnica. 
Flores, C. (2019). Las competencias digitales en los alumnos de ingeniería agronómica de la universidad nacional de cuyo. (Licenciatura en Tecnología Educativa Tesina), Universidad Tecnológica Nacional. Facultad Regional Mendoza, Ecuador.

Freire, P. (1995). La educación como una acción cultural. San José, Costa Rica: EUNED.

Galano, C., Curi, M., Motomura, O. et al. (2002). "Manifiesto por la vida: Por una ética para la sustentabilidad". Ambiente \& Sociedade, V(10).

Gómez, E., Fernando, D., Aponte, G. y Betancourt, L. (2014). "Metodología para la revisión bibliográfica y la gestión de información de temas científicos, a través de su estructuración y sistematización". Revista Dyna, 81(184), 158-163.

Martínez, R. (2010). "La importancia de la educación ambiental ante la problemática actual". Revista Electrónica Educare, XIV(1), 97-111.

Moncada, S. (2014). "Cómo realizar una búsqueda de información eficiente. Foco en estudiantes, profesores e investigadores en el área educativa". Revista Investigación en Educación Médica, 3(10), 106-115.

Reyes, J., Cárdena, M. y Gavilánez, T. C. (2020). "Desarrollo de competencias investigativas mediadas por tecnologías en estudiantes de la carrera de agronomía". Revista Conrado, 16(73), 108-113.

Rojas, E., Quintero, J. y Ancízar, R. (2003). " Investigación pedagógica en el currículo de educación ambiental en la universidad de caldas colombia". Revista Iberoamericana de Educación(11), 112.

Sarango, J. A., Sánchez, S. y Landívar, J. (2016). "Educación ambiental. ¿por qué la historia?". Revista Universidad y Sociedad, 8(3), 184 -187.

Secretaría de Ambiente y Desarrollo Sustentable. (2009). Educación ambiental. Aportes políticos y pedagógicos en la construcción del campo de la educación ambiental. Ciudad Autónoma de Buenos Aires, República Argentina: Secretaría de Ambiente y Desarrollo Sustentable de la Nación. 
UNESCO. (2004). Education for a sustainable development. Fecha de consulta 1-8-2020, 2020, Disponible en: http://portal.unesco.org.education/en/ev.phpURL ID $=23298 \&$ URL DO $=$ DO TOPIC\&URLSECTION=201html

UNESCO. (2014). Década por una educación para la sostenibilidad: Organización de Estados Iberoamericanos.

UNESCO Etxea. (2020). Manual de educación para la sostenibilidad: UNESCO.

Wilson, V., Rodríguez, M., Jiménez, J. y Nieves, M. (2013). "El uso inteligente de herramientas de búsqueda". Perfiles Educativos, XXXV(141), 115-130. 